\title{
Uso problemático de internet en adolescentes: factores personales de riesgo y consecuencias emocionales y conductuales.
}

\author{
Cristina Alonso*1 y Estrella Romero². \\ 1 Institut d'Assistència Sanitària (IAS) \\ 2 Universidad de Santiago de Compostela (USC) \\ - Recibido: 29 - 01 - 2021 . Aceptado: 24 - 03 - 2021 . Avance online: 26 - 04 - 2021
}

RESUMEN. Antecedentes/Objetivo: El objetivo de este estudio es analizar si los cinco grandes de personalidad son predictores del uso problemático de internet (UPI) y si éste predice cambios en el bienestar emocional y en las conductas externalizantes, así como en las conductas de riesgo asociadas a internet (ciberacoso y sexting). Método: Se analizan los datos de 624 adolescentes estudiados en dos ocasiones (T1 y T2) distanciadas por un período de un año, y fueron evaluados el UPI así como los cinco grandes de personalidad y las potenciales consecuencias. Resultados: Los resultados muestran que una baja responsabilidad predice aumento del UPI entre T1 y T2. Además, los resultados ponen de manifiesto que un alto UPI predice aumentos en la conducta agresiva tanto proactiva como reactiva y en el consumo de sustancias. Una alta puntuación en UPI predice descenso de las emociones positivas. Los resultados también sugieren que un alto UPI predice aumento de cibervictimización y ciberagresión. Conclusiones: Este estudio permite comprobar que la personalidad predice el desarrollo del UPI en la adolescencia y que el UPI contribuye al desarrollo de problemas externalizantes y al deterioro de bienestar subjetivo.

PALABRAS CLAVE: Uso problemático de internet, Cinco grandes de personalidad, Consecuencias psicológicas, Adolescentes, Estudio longitudinal.

Problematic internet use in adolescents: personal risk factors and emotional and behavioral outcomes.

ABSTRACT. Background/Objective: The aim of this study is to analyze whether the big five personality traits are predictors of problematic internet use (PIU) and whether PIU predicts changes in emotional well-being and externalizing behaviors, as well as in risk internet-related behaviors (cyberbullying and sexting). Method: This study analysed data from 624 adolescents studied on two occasions (T1 and T2) separated by a one-year period, with evaluations of the big five traits, PIU, and the potential outcomes. Results: The results show that low conscientiousness predicts increases in PIU from T1 to T2. Furthermore, results show that a high PIU predicts increases in both proactive and reactive aggressive behavior and in substance use. A high PIU score predicts a decrease in positive emotions. The results also suggest that a high PIU predicts increased cybervictimization and cyberbullying. Conclusions: This study makes it possible to verify that personality predicts the development of PIU in adolescence and that PIU contributes to the development of externalizing problems and the deterioration of subjective well-being.

KEYWORDS: Problematic internet use, Big five personality, Psychological outcomes, Adolescents, Longitudinal study.

Las tecnologías de la información y la comunicación (TIC) se han incorporado a la vida cotidiana de las personas, ocasionando cambios

*Correspondencia: Cristina Alonso Vilar

Centre de Salut Mental

Dirección: C/ Sant Ignasi, 27, 17430. Santa Coloma de Farners,

Girona (España)

E-mail: cristina.alonso@ias.cat

(C) 2021 Sociedad Universitaria de Investigación en Psicologíay Salud. Publicado por Consejo General de Colegios Oficiales de Psicólogos, España. Este es un artículo Open Access
bajo la CC BY-NC-ND licencia (http://creativecommons.org/licencias/by-nc-nd/4.0/).

Citar como/Cite as: Alonso, C. y Romero, E. (2021). Uso problemático de internet en adolescentes: factores personales de riesgo y consecuencias emocionales $y$ conductuales. Revista Iberoamericana de Psicología y Salud, 12(2), 76-89. https://doi org/10.23923/i.rips.2021.01.046 significativos en los estilos de comportamiento y de relación con los demás. Sin embargo, la amplia difusión de las TIC ha conllevado la aparición de desajustes comportamentales tales como el uso problemático de internet (UPI), a los que son particularmente vulnerables los adolescentes. El UPI se define como una preocupación desadaptativa por el uso de internet que produce angustia o funcionamiento 
disfuncional en las áreas básicas de la vida de los menores (Shapira et al., 2000). A pesar de la enorme variabilidad en los resultados de prevalencias en los estudios previos, se han encontrado altas cifras de prevalencia del UPI con rangos entre un $4,4 \%$ de UPI grave (Durkee et al., 2012) y un $50,2 \%$ de UPI moderado (Alonso y Romero, 2018). Los estudios previos coinciden en señalar la preocupación por el auge de esta problemática y la necesidad de que tanto profesionales sanitarios como de la educación actúen coordinadamente para prevenir e intervenir en el uso inadecuado que los adolescentes realizan de internet (Echeburúa y De Corral, 2010; Fandiño-Leguia, 2017).

La personalidad es uno de los ámbitos de funcionamiento psicológico que más se ha relacionado con el UPI (Weibel et al., 2010). Variables de personalidad como la ansiedad, la ansiedad social, la depresión (e.g. Anderson et al., 2016; Gámez-Guadix, 2014), la hostilidad (Stavropoulos et al., 2017) o el autocontrol y la impulsividad (Gámez-Guadix y Villa-George, 2015; Hong et al., 2014) se han encontrado en la literatura previa como predictores del UPI. En general, la literatura científica previa ha analizado distintas variables de personalidad de forma aislada pero se hace necesario analizar la personalidad desde modelos más integradores. Particularmente, el Modelo de los Cinco Grandes (MCG; McCrae y Costa, 1987) es un esquema léxico-factorial cuya estructura se ha corroborado en múltiples lenguas y culturas; además, en la actualidad es uno de los esquemas clasificadores de los rasgos de personalidad más influyentes (e.g. Andreassen et al., 2013). Dicho modelo propone que las dimensiones de personalidad se aglutinan bajo cinco grandes rasgos: neuroticismo, extraversión, apertura a la experiencia, amabilidad y responsabilidad. Numerosos estudios han constatado la relación entre el UPI y el MCG. En concreto, un metaanálisis (Kayis et al., 2016) informa de relaciones significativas del UPI con las cinco dimensiones del MCG: asociaciones negativas con la extraversión, la responsabilidad, la amabilidad, la apertura y asociación positiva con el neuroticismo. Estudios posteriores confirman que la extraversión, la responsabilidad, la apertura y el neuroticismo están relacionados con el UPI tanto en muestras clínicas como comunitarias (e.g., Alonso y Romero, 2017, 2018; Koporcic y Rucevic, 2018).

Sin embargo, escasos estudios han analizado con datos longitudinales el poder de los cinco grandes de personalidad para predecir, prospectivamente, el UPI, es decir, escasos estudios han clarificado en qué medida los rasgos de personalidad permiten predecir a corto-medio plazo cambios en el UPI. Estos estudios encuentran congruencia con relación a la dimensión de neuroticismo (alto neuroticismo predice un aumento de UPI) (Roma et al., 2019; Thorsteinsson y Davey, 2014). Sin embargo, los resultados son diversos en relación con otras dimensiones predictoras del UPI: extraversión (Thorsteinsson y Davey, 2014; Xiao et al., 2019), amabilidad (Roma et al., 2019) y responsabilidad (Xiao et al., 2019).Por lo tanto, se necesitan más estudios que clarifiquen el valor de las dimensiones del MCG como predictores del UPI.

Dentro del estudio del UPI, otras líneas de trabajo se han centrado en las consecuencias psicosociales que este fenómeno puede acarrear entre los adolescentes. Particularmente, algunos estudios sobre consecuencias emocionales han encontrado que el UPI se asocia a una menor satisfacción con la vida (e.g. Wang et al., 2016) y encuentran que tiene un impacto directo sobre la ansiedad y la depresión (Akin y Iskender, 2011 ; Estévez et al., 2017). Una asociación repetidamente considerada en el UPI es la baja autoestima (Cheung et al., 2018), que puede contribuir a que los adolescentes tengan poca valoración y poca confianza de sí mismos, así como inseguridad en sus habilidades y atributos personales. Otros estudios están más enfocados en conductas externalizantes; por ejemplo, varios estudios han encontrado altos niveles de agresión asociados al UPI (Obeid et al., 2019; Prabakaran y Venkatachalam, 2020). Los estudios sobre el comportamiento agresivo han concluido que existen diferentes tipos de agresión, que pueden tener importantes y diferenciales implicaciones en el funcionamiento de los adolescentes (Carroll et al., 2018). Dodge y Coie (1987) clasificaron la agresividad desde un punto de vista motivacional y funcional, y distinguen dos tipos de agresión: reactiva y proactiva. La 
agresión reactiva se trata de un comportamiento que sucede como reacción a una amenaza percibida y que suele estar relacionada con una activación emocional intensa, altos niveles de impulsividad y hostilidad. Mientras, la agresión proactiva es una agresión fría, instrumental y organizada, que no requiere de la activación con la que está caracterizada la agresión reactiva y surge para dañar a otros con fines de ganancia secundarias. Esta clasificación de conducta agresiva ha sido analizada en relación a múltiples problemas de conducta (Vitaro et al., 2006) por lo que parece que también puede tener una implicación significativa en la conducta de UPI. Sin embargo, escasos estudios han analizado la agresividad proactiva/reactiva en relación al UPI; los primeros resultados apuntan a una mayor relación del UPI con la agresividad proactiva (Little et al., 2003; TurPorcar et al., 2019). Además de la conducta agresiva, otra conducta externalizante que se ha asociado repetidamente con el UPI es el consumo de sustancias, encontrando una mayor probabilidad de consumir tabaco, alcohol y cannabis (e.g. Eraso y Fernández, 2020). Algunos estudios han analizado desde una perspectiva longitudinal estos resultados encontrando que el UPI predice a largo plazo consumo problemático de alcohol (GámezGuadix et al., 2015; Shaffer et al., 2004). Y por último, cabe señalar que el UPI puede conllevar la implicación de los adolescentes en otros comportamientos problemáticos donde internet es una pieza clave como puede ser el ciberacoso y el sexting. De hecho, algunos estudios apuntan en esta dirección encontrando relaciones significativas entre el UPI y el ciberacoso (Arpaci et al., 2020; Stodt, Wegmann y Brand, 2016) y entre el UPI y el sexting (Gómez-García et al., 2020).

Aunque ha habido interés por delimitar las posibles consecuencias psicosociales del UPI, se necesita conocer si el UPI realmente predice cambios emocionales y/o conductuales en los adolescentes, y para ello se requiere un diseño longitudinal.

Partiendo de estas consideraciones, el presente estudio intenta paliar esta carencia en la investigación previa; por ello los objetivos de estudio son 1) analizar si los cinco grandes rasgos de personalidad son predictores significativos del UPI en el plazo de seguimiento de un año y 2) examinar si el UPI predice cambios en el bienestar emocional, en las conductas externalizantes así como en las conductas de riesgo asociadas a internet (ciberacoso y sexting) en un año de seguimiento.

De acuerdo con la literatura previa, y teniendo en cuenta los escasos estudios longitudinales existentes, podría esperarse que el neuroticismo será la dimensión del MCG que surja como predictora del UPI (Roma et al., 2019; Thorsteinsson y Davey, 2014). Además, basándonos en los resultados de estudios transversales, se puede esperar que el UPI, dentro de las variables de bienestar emocional, acarree un descenso de la satisfacción con la vida (Wang et al., 2016) y baja autoestima (Cheung et al., 2018). Además, teniendo en cuenta las conductas externalizantes, se podría esperar que conlleve aumentos en la conducta agresiva, en concreto, en la conducta agresiva proactiva (Little et al., 2013; Tur-Porcar et al., 2019) y aumentos en el consumo de sustancias (Gámez-Guadix et al., 2015). Por último, los adolescentes con un UPI probablemente tendrán una mayor probabilidad de mostrar aumentos en las conductas de ciberacoso y sexting (Arpaci et al., 2020; Gómez-García et al., 2020).

\section{MÉTODO}

\section{- MUESTRA}

La muestra está compuesta por 624 adolescentes que se han evaluado en dos tiempos separados por un año. La evaluación se inició en el curso académico 2015/2016 (T1) y la segunda medición se realizó un año más tarde, en el curso académico 2016/2017 (T2). Los adolescentes estaban escolarizados en 8 centros educativos gallegos y pertenecían a diversos cursos de la Enseñanza Secundaria Obligatoria y de Bachillerato, en centros públicos tanto de zonas urbanas como semiurbanas. Un 55\% de los adolescentes que componen la muestra son chicas y la edad media de los participantes es de 14.35, con una desviación típica de 1.55 (rango de edades entre 12 y 19). 


\section{-INSTRUMENTOS}

\section{MEDIDAS ADMINISTRADAS EN T1}

Cinco grandes rasgos de personalidad. Para evaluar los rasgos de personalidad se ha utilizado el JS NEO-S (Ortet et al., 2010), compuesto por 150 ítems que se contestan con un formato tipo Likert de 5 puntos desde 1 -totalmente en desacuerdo a 5-totalmente de acuerdo. Este cuestionario mide los cinco grandes rasgos de personalidad en los adolescentes: neuroticismo (e.g. "Soy una persona tranquila"), extraversión (e.g. "Soy una persona alegre y animada"), apertura (e.g. "Tengo mucha fantasía"), amabilidad (e.g. "Me parece que la mayoría de la gente con la que trato es honrada y digna de confianza") y responsabilidad (e.g. "Trato de realizar a conciencia todas las cosas que se me encargan"). EI JS NEO-S fue desarrollado como una adaptación del NEO PI-R para jóvenes (Ortet et al., 2012), y tanto la versión breve como completa han demostrado adecuadas propiedades psicométricas en estudios previos (Ortet et al., 2010). En el presente estudio, los coeficientes alfa para las cinco dimensiones son las siguientes: neuroticismo .87, extraversión .86, apertura .81 , amabilidad .82 y responsabilidad .90.

\section{MEDIDAS ADMINISTRADAS EN T1 Y T2}

UPI. Para evaluar el uso de internet se ha utilizado el Internet Addiction Test (IAT, Young, 1998). Consta de 20 ítems que se contestan en una escala de 1-nunca o rara vez a 5-siempre (e.g. "¿Con qué frecuencia Internet te ayuda a evadirte, a bloquear pensamientos sobre tu vida que te intranquilizan?"). Este instrumento permite obtener una puntuación global del UPI. En nuestro estudio se han obtenido unos coeficientes alfa en T1 de .91 y en T2 de .89.

BIENESTAR EMOCIONAL. Para evaluar el bienestar emocional se utilizaron dos instrumentos:

- Escala de Afecto Positivo y Negativo (PANAS; Watson, Clark y Tellegen, 1988) que incluye 20 ítems, 10 de los cuales correspondientes a la subescala de Afecto Positivo (e.g. "Entusiasmada", "Orgulloso") y 10 a la subescala de Afecto Negativo (e.g. "Irritable", "Con miedo"); el período de referencia utilizado fue el último año. En nuestro estudio se han obtenido unos coeficientes alfa en $\mathrm{Tl}$ de .87 para afecto positivo y .88 para afecto negativo y unos coeficientes alfa en T2 de .85 para afecto positivo y de .88 para afecto negativo.

- Escala de Autoestima (EAR; Rosenberg, 1965) que consta de 10 ítems que se contestan en una escala de cuatro puntos (entre $1=$ Muy de acuerdo y $4=$ Muy en desacuerdo), referidos a la valoración y aceptación que el adolescente hace y que acostumbra a mantener con respecto a sí mismo (e.g. "Estoy convencido/a de que tengo buenas cualidades", "Tengo una actitud positiva hacia mí mismo/a"). El coeficiente alfa obtenido en este estudio tanto en T1 como en T2 es de .84.

CONDUCTAS EXTERNALIZANTES. Para evaluar las conductas externalizantes (conducta agresiva y consumo de sustancias) se utilizaron dos instrumentos:

- Cuestionario de agresión proactivareactiva (RPQ; Raine et al., 2006; adaptación española de Andreu, Peña y Ramírez, 2009) que consta de 23 afirmaciones con una escala de respuesta entre $0=$ nunca y $2=$ a menudo, que recogen distintas conductas agresivas: 12 proactivas (e.g. "Has gritado a otros para aprovecharte de ellos") y 11 reactivas (e.g. "Te has enfadado cuando otros te han amenazado"). En nuestro estudio se han obtenido unos coeficientes alfa para la agresión proactiva de .87 en $\mathrm{T} 1$ y .83 en $\mathrm{T} 2$ y de .84 en $\mathrm{T} 1$ y .83 en T2 para la agresión reactiva.

- Cuestionario de consumo de drogas (CCD; Luengo, Otero, Mirón y Romero, 1995) que está compuesto por tres ítems que evalúan el consumo mensual de tabaco, alcohol y cannabis con un formato de respuesta entre $0=$ nunca y $5=$ más de 20 días al mes (e.g. "¿Cuántos días has fumado cigarrillos en el último mes?") 
CONDUCTAS DE RIESGO ASOCIADAS A INTERNET. Se evaluaron dos conductas de riesgo asociadas a internet, esto es, el ciberacoso y el sexting.

- Para evaluar el ciberacoso se utilizó la versión española del European Cyberbullying Intervention Project Questionnaire (ECIPQ; Del Rey et al., 2015) que consta de 22 ítems que, en este trabajo, se utilizaron con una escala de respuesta de Nunca-0 veces (0), Pocas vecesEntre 1 y 2 veces (1), Algunas veces-Entre 3 y 5 veces (2), Bastantes veces-Entre 6 y 10 veces (3) y Muchas veces-Más de 10 veces (4), tomando como referencia un intervalo de tiempo de los últimos seis meses. El cuestionario consta de dos dimensiones: cibervictimización y ciberagresión. Tanto para ciberagresión como para cibervictimización, los ítems hacen referencia a acciones como decir palabras malsonantes, excluir o difundir rumores, suplantar la identidad, etc. en medios electrónicos (e.g. "Alguien ha colgado información personal sobre mí en internet", "He excluido o ignorado a alguien en una red social o chat"). Los coeficientes alfa en T1 son de .87 en la dimensión de cibervictimización y de .85 en la dimensión de ciberagresión. Así mismo, en T2, los coeficientes son de .85 y .86 respectivamente.

- Para evaluar el sexting se ha utilizado el Cuestionario Frequency of Sexting (Weisskirch y Delevi, 2011) que consta de 5 ítems tipo Likert de cinco opciones de respuesta, con una puntuación entre 0 (Nunca) y 4 (Con frecuencia). Los ítems hacían referencia a enviar una foto o un video sexy de uno mismo, en ropa interior, desnudos, enviar mensajes de texto (WhatsApp, SMS, etc.) sexualmente provocativos o con la intención de mantener algún tipo de relación sexual (e.g. "¿Cuántas veces has enviado, a través de teléfono móvil, una foto o vídeo desnudo de ti mismo?"). En este estudio se ha obtenido un alfa de .84 tanto en T1 como en T2.

\section{-PROCEDIMIENTO}

Se estableció contacto con 14 centros educativos de Galicia, de los cuales 8 accedieron a participar en este estudio. Los cuestionarios fueron cumplimentados por los adolescentes durante una sesión lectiva (50 minutos), en las aulas de los centros educativos entre los meses de octubre de 2015 y febrero de 2016 en T1 y entre los meses de octubre 2016 y febrero de 2017 en T2, bajo supervisión de un miembro del equipo investigador, y después de obtener el consentimiento parental y de los propios adolescentes. Se garantizó el anonimato y la confidencialidad de los datos recogidos a través de una clave autogenerada por los adolescentes con la que se pudo emparejar los cuestionarios de la misma persona en T1 y T2.

\section{-ANÁLISIS DE DATOS}

Para responder al primer objetivo del estudio (analizar si los cinco grandes rasgos de personalidad son predictores significativos del UPI en un año de seguimiento), se realizó un análisis de regresión jerárquica tomando el UPI T2 como variable criterio. Dado que previamente se ha encontrado que el UPI correlaciona significativamente con la edad (.08; $p<.05$; Alonso y Romero, 2018) y que también se asocia con el género $(t=3.66$, $881, p<.05$; media de 44.46 en chicos y de 41.01 en chicas; Alonso y Romero, 2018), se incluyeron esas variables en el primer paso de la ecuación; para la variable género se derivó una variable dummy considerando $0=$ chico y $1=$ chica. En un segundo paso se incluyó el UPI evaluado en T1 (efectos autorregresivos; Selig y Little, 2012) y, finalmente, se incluyeron los cinco grandes rasgos de personalidad. De este modo, se examina en qué medida los rasgos contribuyen a predecir el cambio en UPI entre T1 y T2.

Para dar respuesta al segundo objetivo del estudio (examinar si el UPI predice cambios en el bienestar emocional, en las conductas externalizantes y en las conductas de riesgo asociadas a internet en el plazo de un año de seguimiento), se realizaron análisis de regresión jerárquica tomando el UPI TI como predictor y las potenciales consecuencias en T2 como variables criterio, una vez controladas las variables sociodemográficas de género y edad y también los niveles de estas variables en $\mathrm{T} 1$. 


\section{RESULTADOS}

Para todos los análisis de regresión realizados, se examinó si podrían existir amenazas derivadas de la colinealidad; en todos los casos los índices de tolerancia fueron mayores a 0,10 y los factores de inflación de la varianza (FIV) tuvieron valores menores a 10, lo que indica que la colinealidad no está afectando sustancialmente a la estimación de los coeficientes ni al poder de los análisis (Hair et al., 2010).

En la Tabla 1 se presentan los resultados del análisis de regresión jerárquica que permite conocer si las variables de personalidad contribuyen a predecir el cambio en el UPI entre $\mathrm{T} 1$ y $\mathrm{T} 2$.

Los resultados del análisis de regresión jerárquico muestran que, en el primer paso, el géneroyla edadhacen unaaportaciónsignificativa a la predicción del UPI T2, en concreto, ser chico y tener más edad predicen altos niveles de UPI en T2; en el segundo paso, el UPI T1 también predice, con una beta significativa y positiva, el UPI T2, lo que indica un grado significativo de estabilidad entre T1 y T2. En el tercer paso de la ecuación, la responsabilidad emerge como predictor significativo del UPI. El signo negativo de la beta indica que la baja responsabilidad predice aumentos en la conducta de UPI a un año de seguimiento. Como es esperable, dado la parcialización del efecto del UPI previo, la varianza explicada por los rasgos es pequeña, aunque significativa.

En las Tablas 2, 3 y 4 se presentan los resultados del análisis de regresión jerárquica que analiza la predicción del cambio en el bienestar emocional, conductas externalizantes y conductas de riesgo asociadas a internet, partiendo del UPI.

Tabla 1

Análisis de regresión jerárquica para la predicción del cambio en UPI entre T1 y T2 partiendo de los cinco grandes rasgos de personalidad.

\begin{tabular}{|c|c|c|c|}
\hline & & \multicolumn{2}{|c|}{ UPI T2 } \\
\hline & & $\beta$ & $\mathrm{R}^{2}$ \\
\hline \multirow[t]{3}{*}{ Paso 1} & & & .03 \\
\hline & Género & $-.13 * * *$ & \\
\hline & Edad & $.12^{\star \star}$ & \\
\hline \multirow[t]{2}{*}{ Paso 2} & & & .34 \\
\hline & UPI T1 & $.56^{\star \star \star}$ & \\
\hline \multirow[t]{6}{*}{ Paso 3} & & & .35 \\
\hline & Neuroticismo & .04 & \\
\hline & Extraversión & -.01 & \\
\hline & Apertura & .07 & \\
\hline & Amabilidad & .06 & \\
\hline & Responsabilidad & $-.10 * \star$ & \\
\hline Nota: ${ }^{*} p<.05^{* *} p<$. & & & \\
\hline
\end{tabular}


Tabla 2

Análisis de regresión jerárquica para la predicción de los cambios en bienestar emocional entre T1 y T2 partiendo del UPI en T1.

\begin{tabular}{|c|c|c|c|c|c|c|c|}
\hline & & \multicolumn{2}{|c|}{ Emociones positivas T2 } & \multicolumn{2}{|c|}{ Emociones negativas T2 } & \multicolumn{2}{|c|}{ Autoestima T2 } \\
\hline & & $\beta$ & $\mathrm{R}^{2}$ & $\beta$ & $\mathrm{R}^{2}$ & $\beta$ & $\mathrm{R}^{2}$ \\
\hline \multirow[t]{3}{*}{ Paso 1} & & & .01 & & .12 & & .03 \\
\hline & Género & -.05 & & $.24 * \star *$ & & $-.15^{\star \star *}$ & \\
\hline & Edad & $-.10 \star \star$ & & $.25^{\star \star *}$ & & $-.08^{*}$ & \\
\hline \multirow[t]{2}{*}{ Paso 2} & & & .20 & & .31 & & .26 \\
\hline & $\begin{array}{l}\text { Indicador } \\
\text { de bienestar } \\
\text { emocional } \\
\text { en } \mathrm{Tl}\end{array}$ & $.44^{\star \star \star}$ & & $.45^{\star \star \star}$ & & $.48^{\star * *}$ & \\
\hline \multirow[t]{2}{*}{ Paso 3} & & & .21 & & .31 & & .26 \\
\hline & UPI T1 & $-.08^{\star}$ & & .01 & & -.06 & \\
\hline \multicolumn{8}{|c|}{ Nota: ${ }^{*} p<.05^{* *} p<.01^{* * *} p<.001$} \\
\hline
\end{tabular}

\section{Tabla 3}

Análisis de regresión jerárquica para la predicción de los cambios en las conductas externalizantes entre T1 y T2 partiendo del UPI en T1.

\begin{tabular}{|c|c|c|c|c|c|c|c|c|c|c|c|}
\hline & & \multicolumn{2}{|c|}{$\begin{array}{c}\text { Agresión } \\
\text { proactiva T2 }\end{array}$} & \multicolumn{2}{|c|}{$\begin{array}{l}\text { Agresión } \\
\text { reactiva T2 }\end{array}$} & \multicolumn{2}{|c|}{$\begin{array}{l}\text { Tabaco (mes) } \\
\text { T2 }\end{array}$} & \multicolumn{2}{|c|}{$\begin{array}{l}\text { Alcohol (mes) } \\
\text { T2 }\end{array}$} & \multicolumn{2}{|c|}{$\begin{array}{c}\text { Cannabis (mes) } \\
\text { T2 }\end{array}$} \\
\hline & & $\beta$ & $\mathrm{R}^{2}$ & $\beta$ & $\mathrm{R}^{2}$ & $\beta$ & $\mathrm{R}^{2}$ & $\beta$ & $\mathrm{R}^{2}$ & $\beta$ & $\mathrm{R}^{2}$ \\
\hline \multirow[t]{3}{*}{ Paso 1} & & & .05 & & .03 & & .03 & & .15 & & .01 \\
\hline & Género & $-.23^{* \star *}$ & & .04 & & .05 & & $-.12^{\star \star}$ & & -.05 & \\
\hline & Edad & .07 & & $.18^{* \star *}$ & & $.17^{\star \star *}$ & & $.38^{\star \star \star}$ & & $.09 *$ & \\
\hline \multirow[t]{2}{*}{ Paso 2} & & & .24 & & .19 & & .44 & & .24 & & .21 \\
\hline & $\begin{array}{c}\text { Conductas } \\
\text { externalizantes } \\
\text { en Tl }\end{array}$ & $.44^{\star \star \star}$ & & $.41^{* \star \star}$ & & $.65^{\star * *}$ & & $.32^{\star \star \star}$ & & $.46^{\star \star \star}$ & \\
\hline \multirow[t]{2}{*}{ Paso 3} & & & .25 & & .21 & & .44 & & .26 & & .22 \\
\hline & UPI TI & $.10^{*}$ & & $.14^{* * *}$ & & $.08^{* *}$ & & $.14^{* * *}$ & & $.08^{*}$ & \\
\hline$:{ }^{*}$ & $p<.01^{* * *} p$ & & & & & & & & & & \\
\hline
\end{tabular}


En relación con el bienestar emocional (Tabla 2), el análisis de regresión no ha mostrado efecto predictivo significativo sobre las variables de emociones negativas y autoestima. Sin embargo, sí se encuentran resultados significativos en la variable de emociones positivas. En concreto, los resultados indican que la edad, a diferencia del género, contribuye significativamente a la predicción de las emociones positivas en T2, en concreto, una mayor edad predice bajos niveles en las emociones positivas en T2.Además, las emociones positivas en $\mathrm{T} 1$ predicen las emociones positivas en T2. Y por último, los resultados encontrados, una vez controladas las variables sociodemográficas así como los niveles previos de emociones positivas (T1), indican que un alto UPI predice descenso de las emociones positivas en un año de seguimiento.

En relación con la Tabla 3 , los resultados del análisis de regresión muestran que todas las conductas externalizantes evaluadas en este estudio predicen cambios significativos en el UPI. En el primer paso, la edad hace una contribución significativa en la predicción de la agresión reactiva y en el consumo de tabaco, alcohol y cannabis (una mayor edad predice altos niveles en estas variables en T2) mientras que la variable de género hace una aportación significativa a la predicción de la agresión proactiva y consumo de alcohol (ser chico predice aumentos en estas variables en T2). En el segundo paso de la ecuación, en todas las conductas externalizantes se obtuvieron efectos de estabilidad significativos entre T1 y T2. Y del tercer paso, se puede concluir que un alto UPI predice aumentos en la conducta agresiva tanto proactiva como reactiva así como aumentos en el consumo de sustancias (tabaco, alcohol y cannabis) en el plazo de un año de seguimiento.

Respecto a la Tabla 4, los resultados de los análisis de regresión indican que el UPI es un predictor significativo del ciberacoso evaluado en T2. En relación con el primer paso, la edad y el género hacen una contribución significativa en la predicción de la ciberagresión, en concreto,

Tabla 4

Análisis de regresión jerárquica para la predicción de los cambios en las conductas de riesgo asociadas a internet entre T1 y T2 partiendo del UPI en T1.

\begin{tabular}{|c|c|c|c|c|c|c|c|}
\hline & & \multicolumn{2}{|c|}{ Cibervictimización T2 } & \multicolumn{2}{|c|}{ Ciberagresión T2 } & \multicolumn{2}{|c|}{ Sexting T2 } \\
\hline & & $\beta$ & $\mathrm{R}^{2}$ & $\beta$ & $\mathrm{R}^{2}$ & $\beta$ & $\mathrm{R}^{2}$ \\
\hline \multirow[t]{3}{*}{ Paso 1} & & & .01 & & .02 & & .12 \\
\hline & Género & .05 & & $.13^{\star \star \star}$ & & $.13^{\star \star \star}$ & \\
\hline & Edad & -.05 & & $-.09 *$ & & $.31^{\star \star \star}$ & \\
\hline \multirow[t]{2}{*}{ Paso 2} & & & .11 & & .11 & & .19 \\
\hline & $\begin{array}{l}\text { Conductas } \\
\text { de riesgo en } \\
\text { internet en } \mathrm{Tl}\end{array}$ & $.33^{* \star *}$ & & $.30 * * *$ & & $.29 * * *$ & \\
\hline \multirow[t]{2}{*}{ Paso 3} & & & .13 & & .13 & & .20 \\
\hline & UPI TI & $.15^{\star \star \star}$ & & $.17^{* \star *}$ & & .07 & \\
\hline
\end{tabular}


ser chica y tener menos edad predice altos niveles de ciberagresión en T2. En la cibervictimización, las variables sociodemográficas no hacen una aportación significativa. En el segundo paso, se encuentra una estabilidad entre la cibervictimización y la ciberagresión evaluadas en T1 y en T2. Y por último, en el tercer paso, los resultados indican que un alto UPI predice aumento de cibervictimización y ciberagresión en un año de seguimiento.

\section{DISCUSIÓN Y CONCLUSIONES}

Este estudio se ha centrado en la conducta de UPI en una muestra de adolescentes evaluados en dos momentos separados por un año, para intentar examinar los predictores de personalidad así como las potenciales consecuencias emocionales y conductuales.

En cuanto al primer objetivo de nuestro estudio (analizar si los cinco grandes rasgos de personalidad son predictores significativos del UPI en el plazo de seguimiento de un año), los resultados obtenidos sobre las dimensiones generales de personalidad encuentran que la baja responsabilidad predice a un año de seguimiento un aumento de UPI. Este resultado concuerda con los resultados transversales y con la literatura científica previa y apoya a los escasos estudios longitudinales sobre personalidad y UPI que existen (e.g. Stavropoulos et al., 2017). Este hallazgo permite reforzar la importancia de la dimensión de la responsabilidad como factor de riesgo o de protección del UPI de igual manera que ya se ha demostrado para otras adicciones a sustancias (e.g. Montag y Reuter, 2015). Los adolescentes más responsables son autodisciplinados, con un sentido del deber, cautelosos y motivados para luchar por el éxito (Costa y McCrae, 1992), por lo que parece que podrían tener un mayor control sobre el uso de internet en su vida cotidiana.

El segundo objetivo de nuestro estudio fue examinar si el UPI predice cambios en el bienestar emocional, en las conductas externalizantes así como en las conductas de riesgo asociadas a internet (ciberacoso y sexting) en un año de seguimiento. Comenzando por el bienestar emocional, los resultados encontrados indican que una alta puntuación en UPI predice descenso de las emociones positivas. Este resultado es congruente con lo encontrado en otras investigaciones longitudinales en las que se ha encontrado que el UPI es predictor de la angustia emocional (Wartberg et al., 2019) y de un empobrecimiento del bienestar personal de los adolescentes (Yu y Shek, 2018), y que establecen que el deterioro del bienestar personal es más una consecuencia del UPI que una causa del mismo. En relación con las conductas externalizantes, los resultados obtenidos muestran que un alto UPI predice aumentos en la conducta agresiva tanto proactiva como reactiva y en el consumo de sustancias (tabaco, alcohol y cannabis). La predicción del UPI sobre un aumento de la conducta agresiva va en concordancia con los resultados encontrados a nivel transversal que indican que la conducta agresiva es una variable muy relacionada con el UPI (Agbaria, 2020; Alonso y Romero, 2017). Las primeras investigaciones en la literatura previa informaban de una relación entre UPI y conducta agresiva proactiva (e.g. TurPorcar et al., 2019); sin embargo, en nuestros resultados longitudinales encontramos que tanto la agresión proactiva como reactiva es una posible consecuencia del UPI en el plazo de seguimiento de un año. La literatura científica ha encontrado que, con frecuencia, ambos tipos de agresión se manifiestan de forma conjunta en la adolescencia (Penado, Andreu y Peña, 2014); y, aquellos adolescentes que manifiestan ambos tipos de comportamientos agresivos, proactivos y reactivos, presentan características más semejantes a las de la agresión tipo proactivo que reactivo, con una elevada presencia de conducta antisocial (Penado et al., 2014). Por tanto, esta mayor aproximación al tipo de agresión proactiva parece ir en congruencia con lo encontrado en la literatura previa. Por otra parte, dentro de las conductas de corte externalizante, un alto UPI predice aumentos en el consumo de sustancias. Este resultado es acorde con lo encontrado en los escasos estudios longitudinales realizados hasta la fecha sobre el efecto del UPI en el consumo problemático de alcohol (GámezGuadix et al., 2015; Shaffer et al., 2004). Como han señalado otros autores (Gámez-Guadix et al., 2015), las consecuencias psicosociales negativas ocasionadas por el abuso de internet, entre las cuales se puede encontrar un deterioro significativo de su vida personal, social, familiar y 
académico, pueden ejercer su impacto sobre el consumo problemático de sustancias a lo largo del tiempo. Y por último, este estudio permite comprobar que el UPI predice un aumento del ciberacoso, tanto de la cibervictimización como de la ciberagresión, en un año de seguimiento. Este resultado es congruente con lo encontrado en estudios transversales (e.g. Arpaci et al., 2020). Nuestro resultado, más allá de las relaciones transversales, sugiere que los adolescentes que pasan más tiempo en internet y que, además, hacen un uso desadaptativo del mismo, podrían estar más expuestos a los riesgos de internet y favorecer a corto-medio plazo una involucración en conductas de ciberacoso.

Entre las implicaciones prácticas de este estudio encontramos que los resultados permiten identificar un patrón personal de mayor riesgo para el UPI, en el que destaca la baja responsabilidad. En este sentido, una de las dimensiones que ya ha sido tenida en cuenta en algunos programas de prevención es justamente la responsabilidad; esto es, se han comenzado a incluir ámbitos de intervención como el autocontrol y la autoeficacia y se han encontrado efectos favorables sobre el UPI (e.g. Yang y Kim, 2018). En general, los resultados encontrados en la literatura científica en torno a la efectividad de los programas preventivos en la reducción del UPI encuentran que sólo educar a los adolescentes sobre las consecuencias negativas del UPI es ineficaz y debe complementarse con estrategias de intervención dirigidas a cambiar actitudes y habilidades (e.g. Soole et al., 2008); por ello, se hace necesario una vez más, que el entorno formativo del adolescente en riesgo de UPI, establezca intervenciones educativas que se dirijan a fomentar las habilidades propias de la dimensión de personalidad más afectada, esto es, la responsabilidad. Además, teniendo en cuenta los resultados de investigaciones sobre como los estilos parentales influyen en el proceso de socialización de los hijos (e.g. Ruiz-Hernández et al., 2019), el fomento de prácticas parentales de afecto, comunicación y promoción de la autonomía (estilo autorizativo) favorecerá que los adolescentes no presenten problemas de conducta externalizantes, como por ejemplo, el UPI. Por otra parte, los resultados sobre dimensiones emocionales y conductuales asociadas al UPI sugieren que las intervenciones dirigidas a los adolescentes con alto UPI podrán contribuir a la promoción de la salud, en concreto, mejorando el bienestar emocional de los adolescentes, previniendo el consumo de drogas y conductas agresivas y limitando que estos adolescentes se involucren en otras conductas de riesgo como el ciberacoso. En general, cabe destacar que un abordaje educativo en materia de TIC es necesario para evitar que los adolescentes pasen de un uso saludable de internet a un uso problemático. Por ello, la inclusión de las familias y profesionales de la salud y de la educación en los programas de prevención es esencial (Vondrackova y Gabrhelik, 2016).

Estos resultados hay que tomarlos teniendo en cuenta una serie de limitaciones. En primer lugar, la evaluación de la muestra se basa en autoinformes y aunque constituyen un método adecuado en la evaluación de la experiencia subjetiva, su uso puede conllevar sesgos. Por otra parte, a pesar de que este estudio cuenta con un diseño longitudinal, el periodo entre evaluaciones es relativamente corto, de un año de duración, por lo que se necesitarían estudios con periodos de evaluación más largos para evaluar de un modo más exhaustivo el impacto que tiene el UPI en la vida de los jóvenes. Además, este estudio se ha centrado en variables personales implicadas en el UPI; sin embargo, otras variables han mostrado que intervienen en este fenómeno y no se han considerado, por ejemplo, variables familiares, escolares y comunitarias, entre otras.

A pesar de estas limitaciones, esta investigación muestra que, efectivamente, cuando seguimos a los chicos a lo largo del tiempo, los que presentan UPI tienden a ver aumentados sus problemas interpersonales (conducta agresiva), a aumentar su implicación en el uso de sustancias y a mostrar un empeoramiento en su ajuste emocional, así como predice un aumento en otro de los patrones conductuales problemáticos asociados a internet como es el ciberacoso. En general, este estudio permite comprobar los riesgos y consecuencias negativas sobre la salud y el bienestar que tiene el UPI en los adolescentes en un periodo de un año de seguimiento.

\section{- Conflicto de intereses.}

Los autores declaran no tener ningún conflicto de intereses. 


\section{REFERENCES}

Agbaria, Q. (2020). Internet addiction and aggression: the mediating roles of self-control and positive affect. International Journal of Mental Health and Addiction, 1-16. https:// doi.org/10.1007/s $11469-019-00220-z$

Akin, A. y İskender, M. (2011). Internet addiction and depression, anxiety and stress. International Online Journal of Educational Sciences, 3(1), 138-148.

Alonso, C. y Romero, E. (2017). El uso problemático de nuevas tecnologías en una muestra clínica de niños y adolescentes. Personalidad y problemas de conducta asociados. Actas Españolas de Psiquiatría, 45(2), 62-70.

Alonso, C. y Romero, E. (2018). Study of the domains and facets of the five-factor model of personality in problematic internet use in adolescents. International Journal of Mental Health and Addiction, 1-12. https://doi. org/10.1007/s $11469-018-9960-2$

Anderson, E., Steen, E. y Stavropulos, V. (2016). Internet use and Problematic Internet Use: A systematic review of longitudinal research trends in adolescence and emergent adulthood, International Journal of Adolescence and Youth, 22(4), 1-25. https:// doi.org/10.1080/02673843.2016.122771 $\underline{6}$

Andreassen, C.S., Griffiths, M.D., Gjertsen, S.R., Krossbakken, E., Kvam, S. y Pallesen, S. (2013). The relationships between behavioral addictions and the five-factor model of personality. Journal of Behavioral Addiction, 2(2), 90-99. https://doi.org/10.1556/ JBA.2.2013.003

Andreu, J.M., Peña, M.E. y Ramírez, J.M. (2009). Cuestionario de agresión reactiva y proactiva: Un instrumento de medida de la agresión en adolescentes. Revista de Psicopatología y Psicología Clínica, 14, 37 49.

Arpaci, I., Abdeliawad, T., Baloğlu, M., Kesici, S. y Mahariq, I. (2020). Mediating effect of internet addiction on the relationship between individualism and cyberbullying: Cross-
Sectional Questionnaire Study. Journal of Medical Internet Research, 22(5):e16210. https://doi.org/10.2196/16210

Carroll, A., McCarthy, M., Houghton, S., Sanders O'Connor, E. y Zadow, C. (2018). Reactive and proactive aggression as meaningful distinctions at the variable and person level in primary school-aged children. Aggressive behavior, 44(5), 431-441. https://doi. org/10.1002/ab.21763

Cheung, J.C., Chan, K.H., Lui, Y., Tsui, M. y Chan, C. (2018). Psychological well-being and adolescents' internet addiction: A schoolbased cross-sectional study in Hong Kong. Child and Adolescent Social Work Journal, 35, 477-487. https://doi.org/10.1007/s10560018-0543-7

Costa, P.T., Jr. y McCrae, R.R. (1992). The Revised NEO Personality Inventory (NEO$\mathrm{PI}-\mathrm{R}$ ) and NEO-Five-Factor Inventory (NEOFFI) professional manual. Psychological Assessment Resources.

Del Rey, R., Casas, J.A., Ortega-Ruiz, R., Schultze-Krumbholz, A., Scheithaver, H., Smith, P. y Plichta, P. (2015). Structural validation and cross-cultural robustness of the European Cyberbullying Intervention Project Questionnaire. Computers in Human Behavior, 50, 141-147. https://doi.org/10.1016/i. chb.2015.03.065

Dodge, K.A. y Coie, J.D. (1987). Socialinformation-processing factors in reactive and proactive aggression in children's peer groups. Journal of Personality and Social Psychology, 53(6), 1146-1158. https://doi. org/10.1037/0022-3514.53.6.1146

Durkee, T., Kaess, M., Carli, V., Parzer, P., Wasserman, C., Floderus B., Apter, A., Balazs, J., Barzilay, S., Bobes, J., Brunner, R., Corcoran, P., Cosman, D., Cotter, P., Despalins, R., Graber, N., Guillemin, F., Haring, C., Kahn, J. P, ... Wasserman, D. (2012). Prevalence of pathological internet use among adolescents in Europe: Demographic and social factors. Addiction, 107, 2210-2222. https://doi. org/10.1111/i.1360-0443.2012.03946.x

Echeburúa, E. y De Corral, P. (2010). Adicción a las nuevas tecnologías y a las redes 
sociales en jóvenes: n nuevo reto. Adicciones, 22, 91-96. Recuperado de: https://www. adicciones.es/index.php/adicciones/article/ view/196/186

Eraso, A. y Fernández, S. (2020). Uso compulsivo de internet y consumos de sustancias entre los adolescentes españoles. Sistema: Revista de Ciencias Sociales, 257, 19-35.

Estévez, A., Jauregui, P., Sánchez-Marcos, I., López-González, H. y Griffiths, M.D. (2017). Attachment and emotion regulation in substance addictions and behavioural addictions. Journal of Behavioral Addictions, 6(4), 534-544. https://doi. org/10.1556/2006.6.2017.086

Fandiño-Leguia, J.D. (2017). Los estudiantes y el uso problemático de internet. Revista Internacional de Estudios en Educación, 17(2), 145-170. https://doi.org/10.37354/ riee.2017.175

Gámez-Guadix, M. (2014). Depressive symptoms and problematic internet use among adolescents: Analysis of the longitudinal relationships from the cognitive-behavioral model. Cyberpsychology, Behavior and Social Networking, 17(11), 714-719. https://doi. org/10.1089/cyber.2014.0226

Gámez-Guadix, M., Calvete, E., Orue, I. y Las Hayas, C. (2015). Problematic internet use and problematic alcohol use from the cognitive-behavioral model: A longitudinal study among adolescents. Addictive Behaviors, 40, 109-114. https://doi.org/10.1016/i. addbeh.2014.09.009

Gámez-Guadix, M. y Villa-George, F. (2015). El modelo cognitivo-conductual de la adicción a Internet: El papel de la depresión y la impulsividad en adolescentes mexicanos. Psicología y Salud, 25, 111 -122. Recuperado de https://psicologiaysalud.uv.mx/index.php/ psicysalud/article/view/1344/2472

Gómez-García, G., Romero-Rodríguez, J.M., Rodríguez-Jiménez, C. y Ramos Navas-Parejo, M. (2020). Sexting among university students: Links to internet addiction and psychological variables. Journal of Drug and Alcohol Research, 9, Article ID 236105, 16 pages. https://doi.org/10.4303/idar/236105
Hair, J.F, Anderson, R.E, Babin, B.J y Black, W.C. (2010). Multivariate data analysis: A global perspective. 7th ed. Upper Saddle River (N.J.): Pearson education.

Hong, S., You, S., Kim, E. y No, U. (2014). A group-based modeling approach to estimating longitudinal trajectories of Korean adolescents' on-line game time. Personality and Individual Differences, 59, 9-15. https:// doi.org/10.1016/i.paid.2013.10.018

Kayis, A.R., Satici, S.A., Yilmaz, M.F., Simsek, D., Ceyhan, E. y Bakioglu, F. (2016). Big five-personality trait and internet addiction: A meta-analytic review. Computers in Human Behavior, 63, 35-40. https://doi. org/10.1016/i.chb.2016.05.012

Koporčić, M. y Ručević, S. (2018). Odnos osobina ličnosti, tipova usamljenosti i ovisnosti o internetu. Medijska Istraživanja: ZnanstvenoStručni Casopis za Novinarstvo i Medije, 24(2), 69-90. https://doi.org/10.22572/mi.24.2.4

Little, T., Henrich, C., Jones, S. y Hawley, P. (2003). Disentangling the "whys" from the "whats" of aggressive behaviour? International Journal of Behavioral Development, 27, 122-133. https://doi. org/10.1080/01650250244000128

Luengo, A., Otero López J.M., Mirón, L. y Romero, A. (1995). Análisis psicosocial del consumo de drogas en los adolescentes gallegos. Xunta de Galicia

McCrae, R.R. y Costa, P.T. (1987). Validation of the five-factor model of personality across instruments and observers. Journal of Personality and Social Psychology, 52(1), 81-90. https://doi.org/10.1037/00223514.52 .1 .81

Montag, C. y Reuter, M. (Eds.). (2015). Studies in neuroscience, psychology and behavioral economics. Internet addiction: Neuroscientific approaches and therapeutical interventions. Springer Science + Business Media. https:// doi.org/10.1007/978-3-319-07242-5

Obeid, S., Saade, S., Haddad, C., Sacre, H., Khansa, W., Al Haii, R., Kheir, N. y Hallit, S. (2019). Internet addiction among lebanese adolescents: The role of self-esteem, anger, depression, anxiety, social anxiety and fear, 
impulsivity, and aggression-a cross-sectional study. The Journal of Nervous and Mental Disease, 207(10), 838-846. https://doi. org/10.1097/NMD.0000000000001034

Ortet, G., Escrivá, P., Ibañez, M.I., Villa, H., Mezquita, L. y Ruipérez, M.A. (2010). Versión corta de la adaptación española del NEOPI-R (JS NEO-S). International Journal of Clinical and Health Psychology, 10, 327-344. Recuperado de http://www.aepc.es/iichp/ articulos pdf/iichp-354.pdf

Ortet, G., Ibañez, M. I., Moya, J., Villa, H., Viruela, A., y Mezquita, L. (2012). Assessing the five factor of personality in adolescents: The Junior Version of the Spanish NEOPI-R. Assessment, 19, 114-130. https://doi. org/10.1177/1073191111410166

Penado, M., Andreu, J.M. y Peña, E. (2014). Agresividad reactiva, proactiva y mixta: Análisis de los factores de riesgo individual. Anuario de Psicología Jurídica, 24, 37-42. https://doi. org/10.1016/i.api.2014.07.012

Prabakaran, S.A. y Venkatachalam, J. (2020). Exploration of internet usage, procrastination and psychological wellbeing of undergraduate students. Journal of $X_{i}^{\prime}$ an University of Architecture \& Tecnology, 12(2), 2961-2966.

Raine, A., Dodge, K., Loeber, R., Gatzke-Kopp, L., Lynam, D., Reynolds, C., Stouthamer-Loeber, M. y Liu, J. (2006). The reactive-proactive aggression questionnaire: Differential correlates of reactive and proactive aggression in adolescent boys. Aggressive Behavior, 32(2), 159-171. https://doi.org/10.1002/ ab.20115

Roma, P., Ricci, F., Kotzalidis, G.D., Guidarelli, B., Pancheri, C., Mazza, C., Cinti, M.E., Burla, F. y Ferracuti, S. (2019). Psychopathology and personality in problematic internet users. Rivista di Psichiatria, 54(1), 24-30. https://doi. org/10.1708/3104.30937

Rosenberg, M. (1965). Society and the adolescent self-image. Princeton University Press.

Ruiz-Hernández, J.A., Moral-Zafra, E., LlorEsteban, B. y Jiménez-Barbero, J.A. (2019). Influence of parental styles and other psychosocial variables on the development of externalizing behaviors in adolescents:
A systematic review. European Journal of Psychology Applied to Legal Context, 11(1), 9-21. https://doi.org/10.5093/ ejpalc2018a 11

Selig, J.P. y Little, T.D. (2012). Autoregressive and cross-lagged panel analysis for longitudinal data. In B. Laursen, T. D. Little y N. A. Card (Eds.), Handbook of developmental research methods (pp. 265-278). New York, NY: The Guilford Press.

Shaffer, H., LaPlante, D., LaBrie, R., Kidman, R., Donato, A., Stanton, M. (2004). Toward a syndrome model of addiction: Multiple expressions, common etiology. Harvard Review of Psychiatry, 12(6), 367-374. https:// doi.org/10.1080/10673220490905705

Shapira, N.A., Goldsmith, T.D., Keek Jr, P.E., Khosla, U.M. y McElroy, S.L. (2000). Psychiatric features of individuals with problematic internet use. Journal of Affective Disorders, 57 (1-3), 267-272.

Soole, D.W., Mazerolle, L. y Rombouts, S. (2008). School-Based Drug Prevention Programs: A Review of What Works. Australian \& New Zealand Journal of Criminology, 47 (2), 259-286. https://doi.org/10.1375/ acri.4 1.2.259

Stavropoulos, V., Kuss, D.J., Griffiths, M.D., Wilson, P. y Motti-Stefanidi, F. (2017). MMORPG gaming and hostility predict Internet Addiction symptoms in adolescents: An empirical multilevel longitudinal study. Addictive Behaviors, 64, 294-300. https:// doi.org/10.1016/i.addbeh.2015.09.001

Stodt, B., Wegmann, E. y Brand, M. (2016). Predicting dysfunctional internet use: The role of age, conscientiousness, and internet literacy in internet addiction and cyberbullying. International Journal of Cyber Behavior, Psychology and Learning (IJCBPL), 6(4), 28-43. https://doi.org/10.4018/ IJCBPL.2016100103

Thorsteinsson, E.B. y Davey, L. (2014). Adolescents' compulsive internet use and depression: A longitudinal study. Open Journal of Depression, 3(1), 13-17. https:// doi.org/10.4236/oid.2014.31005

Tur-Porcar, A.M., Doménech, A. y Jiménez, 
J. (2019). Eficacia académica percibida, crianza, uso de internet y comportamiento en la adolescencia. Revista Latinoamericana de Psicología, 51(1), 38-47. https://doi. org/10.14349/rlp.2019.v51.n1.5

Vitaro, F., Brendgen, M. y Barker, E.D. (2006). Subtypes of aggressive behaviors: A developmental perspective. International Journal of Behavioral Development, 30(1), 12-19. https://doi. org/10.1177/0165025406059968

Vondrackova, P. y Gabrhelik, R. (2016). Prevention of Internet addiction: A systematic review. Journal of Behavioral Addictions, 5(4), 568-579. https://doi. org/10.1556/2006.5.2016.085

Wang, C.W., Ho, R.T., Chan, C.L. y Tse, S. (2015). Exploring personality characteristics of Chinese adolescents with Internet-related addictive behaviours: Trait differences for gaming addiction and social networking addiction. Addictive Behaviours, 42, 32-35.

Wartberg, L., Kriston, L., Zieglmeier, M., Lincoln, T. y Kammerl, R. (2019). A longitudinal study on psychosocial causes and consequences of Internet gaming disorder in adolescence. Psychological Medicine, 49(2), 287-294.

https://doi.org/10.1017/ S003329171800082X

Watson, D., Clark, L.A., y Tellegen, A. (1988). Development and validation of brief measures of positive and negative affect: The PANAS scales. Journal of Personality and Social Psychology, 54, 1063-1070.

Weibel, D., Wissmath, B. y Groner, R. (2010). Motives for creating a private website and personality of personal homepage owners in terms of extraversion and heuristic orientation. Cyberpsychology: Journal of Psychosocial Research on Cyberspace, 4(1), Article 5. Recuperado de https://cyberpsychology.eu/ article/view/4234/3278

Weisskirch, R.S. y Delevi, R. (2011). "Sexting" and adult romantic attachment. Computers in Human Behavior, 27(5), 1697-1701. https:// doi.org/10.1016/i.chb.2011.02.008

Xiao, J., Li, D., Jia, J., Wang, Y., Sun, W. y Li, D. (2019). The role of stressful life events and the Big Five personality traits in adolescent trajectories of problematic Internet use. Psychology of Addictive Behaviors, 33(4), 360370. https://doi.org/10.1037/adb0000466 Yang, S.Y. y Kim, H.S. (2018). Effects of a prevention program for internet addiction among middle school students in South Korea. Public Health Nursing, 35(3), 246255. hitps://doi.org/10.1111/phn.12394

Young, K.S. (1998). Internet addiction: The emergence of a new clinical disorder. CyberPsychology \& Behavior, 1, 237-244. https://doi.org/10.1089/cpb.1998.1.237

Yu, L. y Shek, D. (2018). Testing longitudinal relationships between internet addiction and well-being in Hong Kong adolescents: Crosslagged analyses based on three waves of data. Child Indicators Research, 17, 15451562. https://doi.org/10.1007/s12187017-9494-3 